\title{
Drug Overdose Leading to Unmasking of Brugada Syndrome
}

\author{
Muhammad Muftia, d, Rajveer Joea ${ }^{\mathrm{a}}$, Vishal Patel ${ }^{\mathrm{a}}$, Mark Lee ${ }^{\mathrm{b}}$, \\ Tanios Maged ${ }^{\mathrm{c}}$, Rashmi Mathew ${ }^{\mathrm{a}}$
}

\begin{abstract}
Brugada syndrome $(\mathrm{BrS})$ is a cardiac autosomal dominant disorder characterized by a constellation of typical electrocardiogram findings as well as clinical criteria including ventricular tachyarrhythmias and sudden death. Although many cases of BrS have been documented, few have identified a drug-induced mechanism as the basis of unmasking the underlying genetic disorder. We present a unique case of a 44-year-old female that was admitted for workup of altered mentation. Upon expanded history, it was evident that multiple psychoactive medications, including amitriptyline, lithium, venlafaxine, and diazepam, taken in duplicity, may have resulted in the observed encephalopathy, and more interestingly, resulted in the unmasking of an underlying BrS with typical electrocardiogram (EKG) changes. Notably, these EKG changes largely reverted to an expected baseline with the cessation of the culprit medications, inferring that drug-induced $\mathrm{BrS}$ is a rare but real entity with important implications. This case illustrates that early identification of susceptible individuals creates opportunity for expedited intervention and strategies to screen for familial inheritance to avert lethal arrhythmias and sudden cardiac death. Moreover, as suspected prescription medications become better studied and characterized, informed decision-making can take place to optimize therapeutic management of psychiatric and cardiac comorbidities.
\end{abstract}

Keywords: Brugada; Brugada syndrome; Amitriptyline; Lithium; Drug overdose

\section{Introduction}

Brugada syndrome $(\mathrm{BrS})$ is an arrhythmogenic cardiac chan-

Manuscript submitted April 22, 2018, accepted May 7, 2018

${ }^{a}$ Department of Medicine, St. Mary Medical Center, Long Beach, CA, USA bDepartment of Electrophysiology, St. Mary Medical Center, Long Beach, CA, USA

${ }^{\mathrm{c}}$ Critical Care Unit, St. Mary Medical Center, Long Beach, CA, USA

${ }^{\mathrm{d} C o r r e s p o n d i n g ~ A u t h o r: ~ M u h a m m a d ~ M u f t i, ~ S t . ~ M a r y ~ M e d i c a l ~ C e n t e r, ~ G M E, ~}$ 1050 Linden Ave., Long Beach, CA 90813, USA.

Email:muneebmufti71@gmail.com

doi: https://doi.org/10.14740/jmc3068w nelopathy characterized by a constellation of electrocardiogram (EKG) changes including a $\geq 2 \mathrm{~mm}$ ST-segment elevation in leads V1 and V2 with a negative T wave and a pseudo right bundle branch block. It is considered a pattern if only EKG changes are seen, and the person is asymptomatic. It is considered a syndrome if it occurs spontaneously or after challenge with intravenous administration of class one antiarrhythmic drugs. We will present a case of a suspected drug overdose which unmasked an underlying BrS.

\section{Case Report}

A 44-year-old female with a history of severe anxiety and depression was brought in by emergency medical services after being found by her parents to be acutely somnolent with slurred speech and altered mentation. Initial suspicion favored accidental overdose of prescription antidepressants and anxiolytics, including amitriptyline, lithium, venlafaxine, and diazepam. Upon further questioning, the patient was able to recall waking up several times throughout the night to take her medications but unable to elucidate how many doses of which medications were taken. On examination, vital signs were notable for hypotension to $90 / 56 \mathrm{~mm} \mathrm{Hg}$, but an otherwise regular heart rate of 97 beats per minute and pulse oximetry of $95 \%$ on room air. Cardiac examination revealed a normal S1 and S2 without additional sounds. The pulmonary exam was clear to auscultation bilaterally and abdominal exam was unremarkable. Neurological exam was notable for alternating directional nystagmus, otherwise non-focal. Initial workup included noncontrast computed tomography (CT) of the head which was negative for an acute intracranial process. EKG done at time of admission (Fig. 1) revealed a first degree AV block with PR interval of $227 \mathrm{~ms}$, a posterior fascicular block, a widened QRS at $121 \mathrm{~ms}, \mathrm{R}^{\prime}$ in aVL, rSR' in V1 and 2 with ST-segment elevation $1 \mathrm{~mm}$ in V1 and $3 \mathrm{~mm}$ in V2 with a coved ST-segment and inverted T wave, as well as a prolonged QTc of 494 ms. Given these initial EKG findings, a further detailed history was obtained including family history. However, the patient and her family denied any known sudden deaths or history of $\mathrm{BrS}$ in the last three generations. A preliminary diagnosis of an accidental prescription drug overdose was made with possible drug-induced BrS. A second repeat EKG was performed to confirm the findings (Fig. 2). No previous EKG could be obtained for the patient. She was given intravenous sodium bi- 


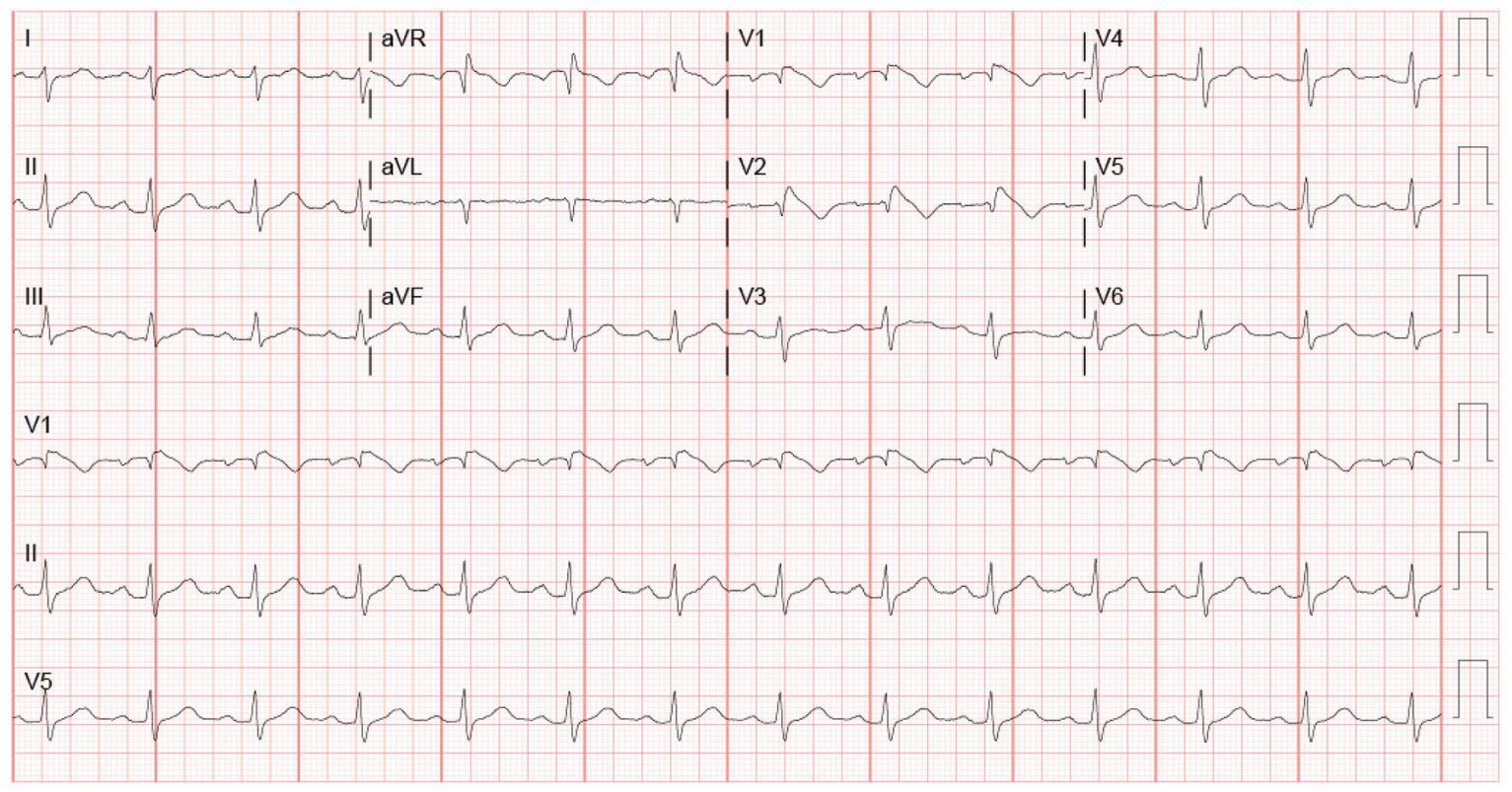

Figure 1. EKG on admission.

carbonate and magnesium given early indications of QTc prolongation. The patient was admitted to the intensive care unit for close monitoring, cardiac telemetry, and serial EKGs. Drug levels were ordered for the patient. Lithium peaked at $1.4 \mathrm{mg} /$ dL. Amitriptyline urine levels were ordered (serum levels not available at our institute) which came out less than $100 \mathrm{ng} / \mathrm{mL}$.

Over the course of next few days, the patient's mental status improved. Transthoracic echocardiography was performed which did not demonstrate structural abnormality or valvular disease. Serial EKGs done showed the return of ST-segment elevations to baseline on hospital day 2 . Before discharge, the pseudo-right bundle branch block with coved ST-segment with nega- tive T wave was still present (Fig. 3). The patient was informed of the final diagnosis and was told to follow-up outpatient with a cardiologist for genetic testing and electrophysiological studies. The patient's primary care and psychiatrist were also informed of the findings to optimize medications accordingly.

\section{Discussion}

Brugada pattern EKG was identified as early as 1953 by Osher and Wolf as right bundle branch block (RBBB) with ST-segment elevation in the right precordial leads. During the 1980s,

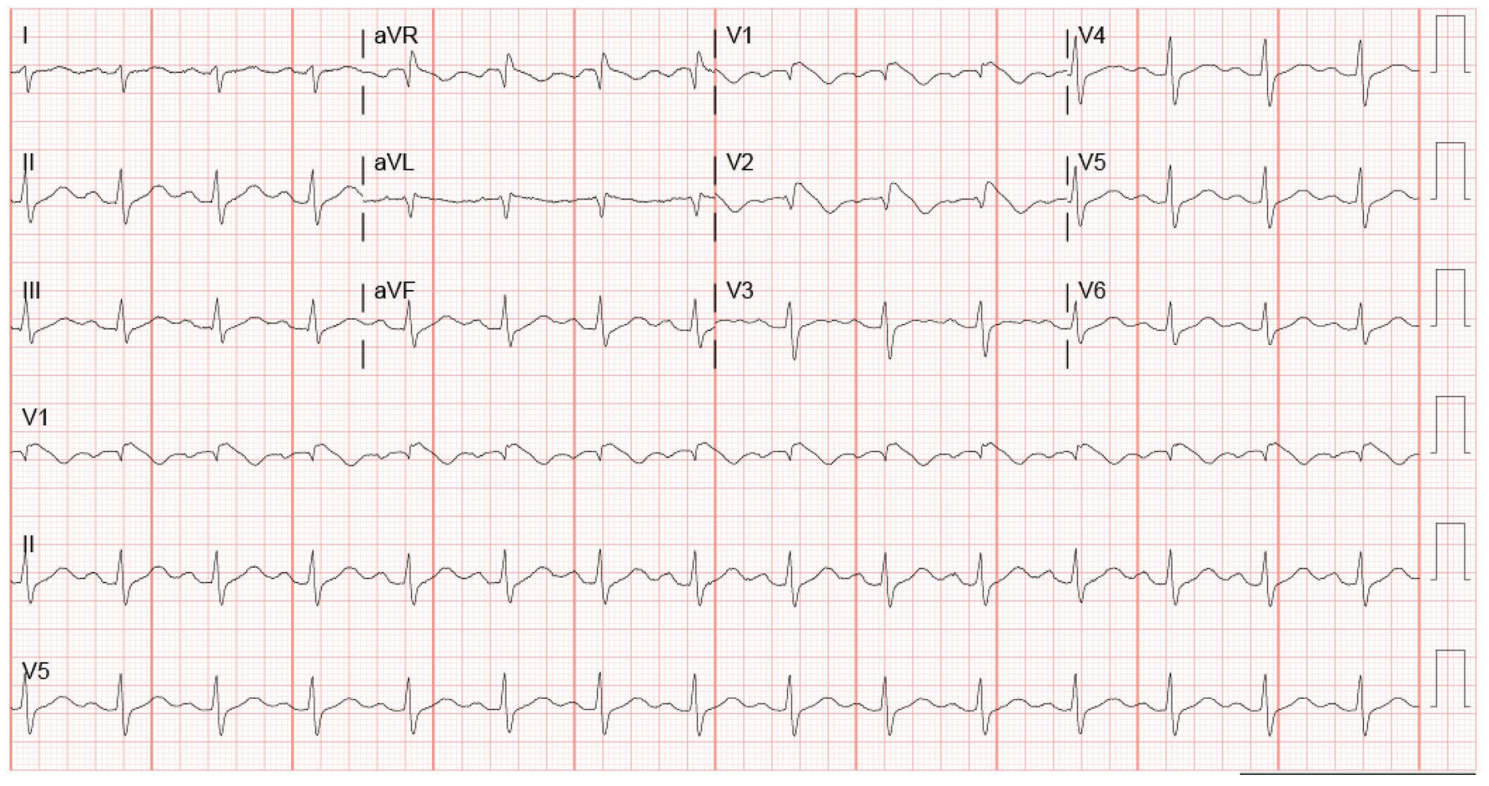

Figure 2. EKG $2 \mathrm{~h}$ after admission. 


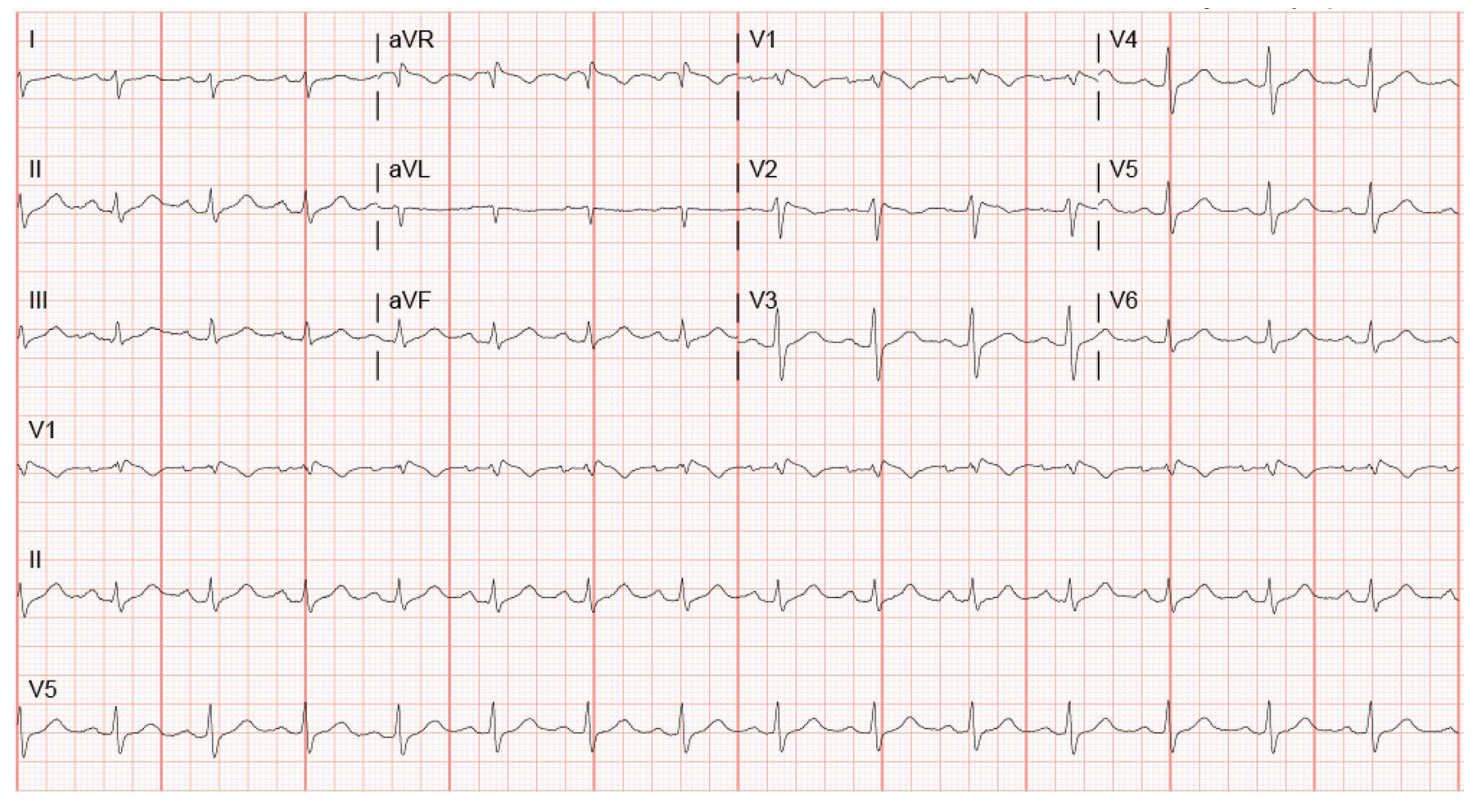

Figure 3. EKG before discharge.

it was noticed that there was an unexpected high number of deaths in immigrants from Thailand which was later classified as sudden unexpected nocturnal death syndrome (SUNDS) [1]. In 1992, Brugada et al presented a study of eight patients with EKG showing RBBB, normal QT interval and persistent ST-segment elevation in precordial leads V1 to V2-V3 [2]. Since the first publication of Brugada entity, multiple cases have been reported in the literature, and different patterns of Brugada in the EKG have been noted. BrS has been associated with sudden cardiac death in otherwise healthy young adults.

BrS is thought to be responsible for causing at least $4 \%$ of all sudden deaths and about $20 \%$ of all deaths with structurally normal hearts [3]. Priori et al showed that Brugada EKG pattern in about two to nine times more likely to occur in men [4]. The youngest patient diagnosed with Brugada was about 2 years old and the oldest 84 years old [3] with the average age for diagnosis being 41 years [5].

The genes involved with BrS have shown autosomal dominant inheritance with a variable expression which includes SCN5A and SCN10A [4]. SCN5A mutation has been seen in about $18-30 \%$ of the families with Brugada [5] and SCN10A in about $17 \%$ of the patients [6]. These genes are located on chromosome $3 \mathrm{p} 22$ [6]. They encode for the sodium channels. The protein encoded from SCN10A has been detected to be in higher levels in the Purkinje system [7]. Some other mutations quoted by other studies include missense mutations in A39V, G490R and S481L were found in patients with short QT $\mathrm{BrS}$ which encode the L-type calcium channel [8]. The exact electrophysiological mechanisms of $\mathrm{BrS}$ are not completely understood, but the suggested mechanisms include early repolarization abnormalities, local conduction abnormalities or local ventricular depolarization [9].

Three types of Brugada pattern EKG have been described. Only type one is diagnostic of BrS while types two and three are only suggestive [3]. Type one has prominent coved ST- segment with a J-point amplitude or ST-segment elevation $\geq$ $2 \mathrm{~mm}$ followed by negative $\mathrm{T}$ wave. Type two has $\geq 2 \mathrm{~mm}$ J-point elevation, $\geq 1 \mathrm{~mm}$ ST-segment elevation with saddleback appearance followed by a positive or biphasic $\mathrm{T}$ wave. Type three has saddleback or coved appearance with $<1 \mathrm{~mm}$ ST-segment elevation.

The most recent expert consensus has defined BrS has type one morphology with $\geq 2 \mathrm{~mm}$ ST-segment elevation in $\geq$ 1 lead in the right precordial leads V1 and V2 either spontaneously or after provocative testing, or having type two or three EKG morphology with $\geq 1$ lead (V1 and V2) which turns into type one EKG after provocative drug test [4].

Drugs overdoses have been linked to BrS which includes tricyclic antidepressants (TCAs), lithium, antihistamines, cocaine, calcium channel blocker and anesthetics like propofol and bupivacaine [9]. Both lithium and amitriptyline are known cardiac sodium channel blockers. Brahmi et al studied 65 patients who had overdosed on TCAs and found that about $15 \%$ showed type one Burgada pattern [10]. Another study of a larger group (402 patients) found that only $2.3 \%$ of the patients had the type one Brugada pattern [11]. Other isolated cases have also shown Brugada pattern leading to ventricular fibrillation and cardiac arrest [12]. Darbar et al described a case of Brugada with lithium plasma concentration of $2.4 \mathrm{mmol} / \mathrm{L}$ and the resolution of the ST elevations after the lithium levels dropped [13]. Littmann et al described a case of EKG findings of Brugada in a patient with recreational cocaine use on multiple admissions, but the procainamide challenge failed to reproduce the sign [14].

Our patient had a history of depression and bipolar disorder and was being given lithium, amitriptyline, venlafaxine, and diazepam. Extensive family history was taken including past three generations, and there was no documented sudden cardiac death history or Brugada pattern recorded. A prior EKG was not available. Our patient's initial EKG was sug- 
gestive of amitriptyline toxicity with first degree AV block, posterior fascicular block, RBBB in V1 and 2 with coved ST segment with elevation, $\mathrm{R}^{\prime}$ in aVR, prolonged QTc suggestive of TCA overdose and type one Brugada. Serial EKGs showed the same pattern. A few days after presentation, the ST elevations returned to baseline in V1-2 but still had the coved STsegment with RBBB.

What makes the situation less clear in our patient is that her lithium peak level recorded at our hospital was 1.4 $\mathrm{mmol} / \mathrm{L}$, and urine amitriptyline levels were less than 100 $\mathrm{ng} / \mathrm{mL}$. The patient could not recall how many extra medication doses she took. Another factor is that her ST-elevations returned to baseline after a couple of days without TCA and lithium, but the ST-segment remained coved pattern. At this point, it remains unclear if the patient has a genetic mutation or if she was susceptible to Brugada morphology and the medications accentuated the pattern. She would require outpatient cardiology follow-up with electrophysiologic studies to assess if arrhythmia can be provoked or reproduced. Another possible follow-up intervention is to implant a loop recorder to monitor for arrhythmias. Finally, a discussion about an automated implantable cardioverter defibrillator (AICD) needs to take place with the patient to discuss the benefits vs. risks. The patient was also advised to reconcile medications and determine appropriate alternatives to TCA and lithium.

\section{Conclusion}

BrS can be unmasked in patients who are being treated with antidepressants including TCA and lithium who are genetically predisposed to it and they are at increased risk of sudden cardiac death due to arrhythmias. If Brugada pattern EKG is seen de novo in a patient taking these medications, BrS should be considered. Risk stratification is an important step in deciding who needs to have AICD placed.

\section{References}

1. Riera AR, Schapachnik E, Ferreira C. Brugada disease: chronology of discovery and paternity. Preliminary observations and historical aspects. Indian Pacing Electrophysiol J. 2003;3(4):253-260.

2. Brugada P, Brugada J. Right bundle branch block, persistent ST segment elevation and sudden cardiac death: a distinct clinical and electrocardiographic syndrome. A multicenter report. J Am Coll Cardiol. 1992;20(6):13911396.

3. Antzelevitch C, Brugada P, Borggrefe M, Brugada J, Bru- gada R, Corrado D, Gussak I, et al. Brugada syndrome: report of the second consensus conference: endorsed by the Heart Rhythm Society and the European Heart Rhythm Association. Circulation. 2005;111(5):659-670.

4. Priori SG, Wilde AA, Horie M, Cho Y, Behr ER, Berul C, Blom N, et al. HRS/EHRA/APHRS expert consensus statement on the diagnosis and management of patients with inherited primary arrhythmia syndromes: document endorsed by HRS, EHRA, and APHRS in May 2013 and by ACCF, AHA, PACES, and AEPC in June 2013. Heart Rhythm. 2013;10(12):1932-1963.

5. Priori SG, Napolitano C, Gasparini M, Pappone C, Della Bella P, Giordano U, Bloise R, et al. Natural history of Brugada syndrome: insights for risk stratification and management. Circulation. 2002;105(11):1342-1347.

6. Hu D, Barajas-Martinez H, Pfeiffer R, Dezi F, Pfeiffer J, Buch T, Betzenhauser MJ, et al. Mutations in SCN10A are responsible for a large fraction of cases of Brugada syndrome. J Am Coll Cardiol. 2014;64(1):66-79.

7. Yang T, Atack TC, Stroud DM, Zhang W, Hall L, Roden DM. Blocking Scn10a channels in heart reduces late sodium current and is antiarrhythmic. Circ Res. 2012;111(3):322-332.

8. Antzelevitch C, Pollevick GD, Cordeiro JM, Casis O, Sanguinetti MC, Aizawa Y, Guerchicoff A, et al. Lossof-function mutations in the cardiac calcium channel underlie a new clinical entity characterized by ST-segment elevation, short QT intervals, and sudden cardiac death. Circulation. 2007;115(4):442-449.

9. Yap YG, Behr ER, Camm AJ. Drug-induced Brugada syndrome. Europace. 2009;11(8):989-994.

10. Brahmi N, Thabet H, Kouraichi N, Driss I, Amamou M. [Brugada syndrome and other cardiovascular abnormalities related to tricyclic antidepressants ans related drugs intoxication]. Arch Mal Coeur Vaiss. 2007;100(1):28-33.

11. Bebarta VS, Phillips S, Eberhardt A, Calihan KJ, Waksman JC, Heard K. Incidence of Brugada electrocardiographic pattern and outcomes of these patients after intentional tricyclic antidepressant ingestion. Am J Cardiol. 2007;100(4):656-660.

12. Tada H, Sticherling C, Oral H, Morady F. Brugada syndrome mimicked by tricyclic antidepressant overdose. J Cardiovasc Electrophysiol. 2001;12(2):275.

13. Crawford RR, Higdon AN, Casey DB, Good DE, Mungrue IN. Multiple lithium-dependent Brugada syndrome unmasking events in a bipolar patient. Clin Case Rep. 2015;3(1):14-18.

14. Littmann L, Monroe MH, Svenson RH. Brugada-type electrocardiographic pattern induced by cocaine. Mayo Clin Proc. 2000;75(8):845-849. 